\title{
Closing the Gap in the Capacity of Wireless Networks Via Percolation Theory
}

\author{
Massimo Franceschetti, Member, IEEE, Olivier Dousse, Member, IEEE, David N. C. Tse, Fellow, IEEE, and \\ Patrick Thiran, Member, IEEE
}

\begin{abstract}
An achievable bit rate per source-destination pair in a wireless network of $n$ randomly located nodes is determined adopting the scaling limit approach of statistical physics. It is shown that randomly scattered nodes can achieve, with high probability, the same $1 / \sqrt{n}$ transmission rate of arbitrarily located nodes. This contrasts with previous results suggesting that a $1 / \sqrt{n \log n}$ reduced rate is the price to pay for the randomness due to the location of the nodes. The network operation strategy to achieve the result corresponds to the transition region between order and disorder of an underlying percolation model. If nodes are allowed to transmit over large distances, then paths of connected nodes that cross the entire network area can be easily found, but these generate excessive interference. If nodes transmit over short distances, then such crossing paths do not exist. Percolation theory ensures that crossing paths form in the transition region between these two extreme scenarios. Nodes along these paths are used as a backbone, relaying data for other nodes, and can transport the total amount of information generated by all the sources. A lower bound on the achievable bit rate is then obtained by performing pairwise coding and decoding at each hop along the paths, and using a time division multiple access scheme.
\end{abstract}

Index Terms-Ad-hoc networks, capacity, percolation theory, scaling laws, throughput, wireless networks.

\section{INTRODUCTION}

W IRELESS networks are modeled as a set of $n$ nodes that exchange messages using radio transmissions. A natural question that arises in such systems is what amount of information the source nodes can send to the destination nodes as the number $n$ grows. The ground breaking work of [10] has shown that, in a network of arbitrarily located nodes, when each node wishes to communicate to a destination located at a nonvanishingly small distance away, the amount of information that can be exchanged by each source-destination pair must go to zero, as $n$ tends to infinity, at least at rate $1 / \sqrt{n}$. This result, originally

Manuscript received October 26, 2004. This work was supported in part by the National Science Foundation, under CAREER award CNS-0546235 and award CCR-0118784; and by the National Competence Center in Research on Mobile Information and Communication Systems (NCCR-MICS), a center supported by the Swiss National Science Foundation by Grant 5005-67322.

M. Franceschetti is with the Department of Electrical and Computer Engineering (ECE), University of California, San Diego, La Jolla, CA 92093 USA (e-mail: massimo@ece.ucsd.edu).

O. Dousse is with the Deutsche Telekom Laboratories, 10587 Berlin, Germany (e-mail: olivier.dousse@ieee.org).

D. N. C. Tse is with the Department of Electrical Engineering and Computer Science (EECS), University of California at Berkeley, Berkeley, CA 94720 USA (e-mail: dtse@eecs.berkeley.edu).

P. Thiran is with the School of Computer and Communication Sciences, EPFL, CH-1015 Lausanne, Switzerland (e-mail: Patrick.Thiran@epfl.ch).

Communicated by G. Sasaki, Associate Editor for Communication Networks. Digital Object Identifier 10.1109/TIT.2006.890791 viewed as a consequence of the interference model used, has been later extended to hold in a more general information theoretic setting [18], assuming only that radio signals are strongly attenuated over distances, following a sufficiently high power decay law, or an exponential attenuation law, which is the typical case if there is absorption in the medium [7].

The authors in [10] have also shown that when nodes are randomly located, each source-destination pair can achieve a bit rate only of order $1 / \sqrt{n \log n}$, by using a specific multihop strategy. Alternative strategies have also been proposed to achieve the same bound [5], [13], [17]. All of these results suggest that a $\sqrt{\log n}$ factor is the price to pay for the randomness due to the location of the nodes. Nevertheless, the information theoretic upper bound of [18] does not preclude a rate higher than $1 / \sqrt{n \log n}$ to be achievable.

This paper shows that a $1 / \sqrt{n}$ rate is achievable in networks of randomly located nodes. Hence, there is no gap between the capacity of randomly located, and arbitrarily located nodes, at least up to scaling and in the high attenuation regime. The constructive strategy that achieves the $1 / \sqrt{n}$ rate is based on multihop transmission, pairwise coding and decoding at each hop, and a time-division multiple-access (TDMA) scheme. The proof of the result follows from percolation theory arguments.

Percolation theory [9], [15] is a field of mathematics and statistical physics that provides models of phase transition phenomena that are observed in nature. It has been used in the past to study connectivity of wireless networks [1], [3], [6], [8], [16], but to the best of our knowledge our paper is the first attempt to exploit percolation to derive information capacity results. We hope that the connection that we establish here can be used in the future to solve other information and network theoretic questions where spatial randomness plays a key role.

To give a glance at the connection we establish in this paper, let us consider the following question originally due to Broadbent and Hammersley to introduce percolation theory [2]. Water is poured on one side of a large (ideally infinite) porous stone. What is the probability that the water finds a path to the opposite side? By modeling the stone as a square grid in which each edge can be open and hence traversed by water with probability $p$, and closed otherwise, independently of all other edges, one can show that for $p>1 / 2$ water percolates trough the stone with probability one. One can then ask at what rate the water percolates and how it depends on $p$. In other words, how rich in disjoint paths is the connected component of open edges? In this paper, we construct a mapping such that the open grid edges of a percolation model correspond to the presence of wireless transmitters in certain locations of the plane, and the open percolating paths represent a wireless backbone that is used to multihop data 
across the network. Accordingly, to maximize the information flow, we want to operate the network at $p>1 / 2$, above the percolation threshold, so that we can guarantee the existence of many disjoint paths that traverse the network, but also have $p<1$, so to avoid overcrowding and excessive interference. We show that controlling the parameter $p$ corresponds to controlling the transmission bit-rate of the nodes as $n \rightarrow \infty$, and we find that the optimal scaling law corresponds to some $1 / 2<p<1$.

Finally, we want to spend few additional words on some related literature. Capacity scaling laws of wireless networks have received enormous attention in the recent literature, see [4], [5], [10], [11], [13], [14], [17], [18] and references therein. Typically, in these works there are two ways of letting the number of nodes $n$ tend to infinity. One can either keep the area where the network is deployed constant, and let the node density $\lambda$ tend to infinity (dense networks); or one can keep the node density $\lambda$ constant, and increase the area to infinity (extended networks). In both of these settings, network theoretic lower bounds on achievable transmission rates can be obtained constructively, for given communication strategies and power attenuation laws; while information theoretic upper bounds must be obtained allowing arbitrary communication strategies and assuming only the power decay law in the propagation medium.

Our lower bound is based on a constructive strategy and holds for extended networks with bounded transmitted and received power, assuming only a power decay law whose tail exhibits a power law behavior with exponent $\alpha>2$, or an exponential attenuation. In the case of dense networks, results hold as long as near field effects of electromagnetic propagation can be neglected. When these come into play, as noted in [4], computed bounds reduce to $1 / n$, and the wireless communication model becomes questionable unless the physical size of the transmitters tends to zero. Our proposed strategy also achieves the optimal average multihop delay required to reach the destination, as defined in [5].

The rest of the paper is organized as follows. In Section II, we summarize the network model and in Section III the main results. In Section IV, we give an overview of our protocol and provide some intuition on why it achieves the optimal transmission rate. In Section V, we formalize the correspondence with percolation theory and show how to construct the wireless backbone. Section VI describes the four phases of the protocol and proves our main results. Section VII is devoted to the dense network case. Finally, Section VIII concludes the paper. Percolation theory results are given in the Appendix.

\section{NETWORK MODEL}

We construct a random extended network by placing nodes according to a Poisson point process of unit intensity on the plane and focus our attention to the square $B_{n}=[0, \sqrt{n}] \times$ $[0, \sqrt{n}]$. Similarly, we construct a dense network by placing nodes according to a Poisson point process of intensity $n$ over a square of unit area. We are mainly concerned with events that occur inside these squares with high probability (w.h.p.); that is, with probability tending to one as $n \rightarrow \infty$.
We pick uniformly at random a matching of source-destination pairs, so that each node is the destination of exactly one source. We assume all nodes transmit at constant power $P$, and that node $x_{j}$ receives the transmitted signal from node $x_{i}$ with power $P \ell\left(x_{i}, x_{j}\right)$, where $\ell\left(x_{i}, x_{j}\right)$ indicates the path loss between $x_{i}$ and $x_{j}$. Being interested only in a lower bound on the achievable rate, we restrict ourselves to a model of communication where the interference at the receiver is simply regarded as noise. Hence, any two nodes can establish a direct communication link, over a channel of unit bandwidth, of rate

$$
R\left(x_{i}, x_{j}\right)=\log \left(1+\frac{P \ell\left(x_{i}, x_{j}\right)}{N_{0}+\sum_{k \neq i} P \ell\left(x_{k}, x_{j}\right)}\right) \mathrm{bit} / \mathrm{s}
$$

where $N_{0}$ is the ambient noise power at the receiver. Our scaling results do not change considering a bandwidth $W \neq 1$, or all nodes transmitting at constant rate $R$, independent of distance, but using different powers for transmission.

The per-node throughput $T(n)$ is defined as the number of bits per second that w.h.p. all nodes can (simultaneously) transmit to their intended destinations. For any source-destination pair, the communication delay $D(n)$ is the number of hops needed to reach the destination, averaged over all network realizations.

Throughout the paper, we use the following probabilistic variation of the order notation. We write $f(n)=O(g(n))$ w.h.p., if there exists a constant $K$ such that

$$
\lim _{n \rightarrow \infty} P(f(n) \leq K g(n))=1 .
$$

We also write $f(n)=\Omega(g(n))$, as $n \rightarrow \infty$, if $g(n)=O(f(n))$ in the sense indicated above.

\section{CONTRIBUTION}

Theorem 1: Let the Euclidean distance between two nodes $x_{i}$ and $x_{j}$ be denoted by $d_{i j}$. Let the power attenuation function be $\ell\left(x_{i}, x_{j}\right)=\min \left\{1, e^{-\gamma d_{i j}} / d_{i j}^{\alpha}\right\}$ with $\gamma>0$, or $\gamma=0$ and $\alpha>2$. We have that

$$
T(n)=\Omega(1 / \sqrt{n}) \mathrm{bit} / \mathrm{s}
$$

is achievable in a random extended network, with a corresponding average number of hops

$$
D(n)=O(\sqrt{n})
$$

Theorem 2: Let the power attenuation function be $\ell\left(x_{i}, x_{j}\right)=e^{-\gamma d_{i j}} / d_{i j}^{\alpha}$ with $\gamma>0$, or $\gamma=0$ and $\alpha>2$. We have that

$$
T(n)=\Omega(1 / \sqrt{n}) \mathrm{bit} / \mathrm{s}
$$

is achievable in a random dense network, with a corresponding average number of hops

$$
D(n)=O(\sqrt{n})
$$




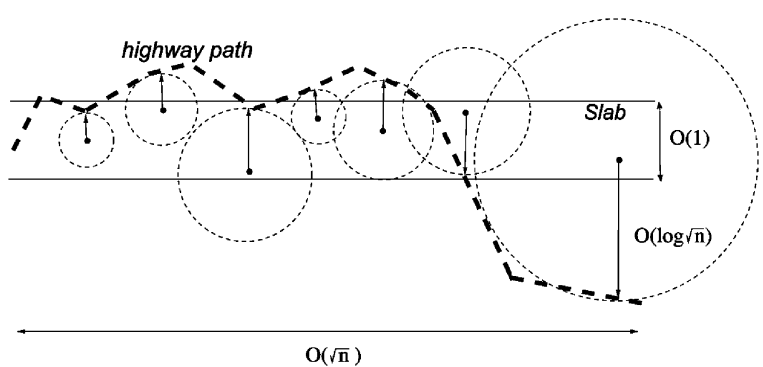

Fig. 1. Nodes inside a slab of constant width access a highway in single hops of length at most proportional to $\log \sqrt{n}$. Multiple hops along the highway are of length bounded above by a constant.

We note that the result of Theorem 2 relies on an ideal power attenuation function that is singular at the origin. In practice, this result holds as long as near field effects are negligible and it is presented for completeness, as it matches the dense network model of [10]. Most of the paper is devoted to the proof of Theorem 1, while Theorem 2 is proven in Section VII, by adapting few steps of the main proof.

\section{OVERVIEW OF THE SOLUTION}

The proposed solution is based on multihop routing, and exploits the formation of paths percolating across the network. We divide the nodes into disjoint sets that cross the network area. These sets form a "highway system" of nodes that can carry information across the network at constant rate, using short hops. The rest of the nodes access the highway system using single hops of longer length. The communication strategy is then divided into four consecutive phases. In a first phase, nodes drain their information to the highway, in a second phase information is carried horizontally across the network through the highway, in a third phase it is carried vertically, and in a last phase information is delivered from the highway to the destination nodes. Fig. 1 shows a schematic representation of the first phase. In each phase we use point-to-point coding and decoding on each Gaussian channel between transmitters and receivers, and design an appropriate time schedule for transmission.

Given the construction outlined above, and letting all nodes transmit with the same power $P$, we might expect the longer hops needed in the first and last phases of the strategy to have a lower bit-rate, due to the higher power loss across longer distances. However, one needs to take into account other components that influence the bit-rate, namely, interference, and relay of information from other nodes. It turns out that when all these components are accounted for, the bottleneck is due to the information carried through the highway.

We now give a short sketch of the proof. First, we notice that the highway system consists of paths of hops whose length is uniformly bounded above by some constant. Then, using a time division protocol, we show that a constant transmission rate can be achieved along each path. However, we also need to account for the relay of information coming from all nodes that access a given path. The number of these nodes is at most proportional to $\sqrt{n}$. This is ensured by associating to each path only those nodes that are within a slab of constant width that crosses the network area, see Fig. 1. It follows that the rate of communication of each node on the highway paths can be of order $1 / \sqrt{n}$.
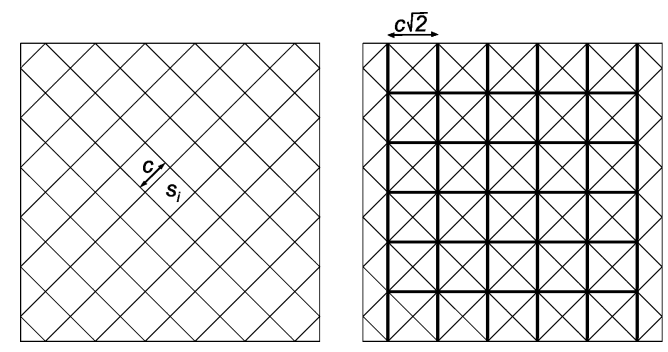

Fig. 2. Construction of the bond percolation model. We declare each square on the left-hand side of the picture open if there is at least a Poisson point inside it, and closed otherwise. This corresponds to associate an edge to each square, traversing it diagonally, as depicted on the right-hand side of the figure, and declare the edge either open or closed according to the state of the corresponding square.

Now, let us consider the rate of the nodes that access the highway in single hops. The proof is completed by showing that these nodes, requiring single hops of length at most proportional to $\log \sqrt{n}$, and not having any relay burden, can sustain a rate higher than $1 / \sqrt{n}$.

Notice that there are three key points in our reasonings: 1) there exist paths of hop length bounded above by a constant that cross the entire network forming the highway system, 2) these paths can be put into a one to one correspondence with $\sqrt{n}$ slabs of constant width, each containing at most a constant times $\sqrt{n}$ number of nodes, and (iii) these paths are somehow regularly spaced so that there is always one within a $\log \sqrt{n}$ distance factor from any node in the network.

In the following, a mapping to a discrete percolation model ensures the existence of many crossing paths. A time division strategy, in conjunction to a counting argument, shows that each path in the highway system can have a constant rate, and that nodes can access the highway at a rate at least proportional to $1 / \sqrt{n}$. Finally, some simple concentration bounds show that the number of nodes that access any given path is at most a constant times $\sqrt{n}$.

\section{THE HighWAY}

To begin our construction, we partition the box $B_{n}$ into subsquares $s_{i}$ of constant side length $c$, as depicted in the left-hand side of Fig. 2. Let $X\left(s_{i}\right)$ be the number of Poisson points inside $s_{i}$. By appropriately choosing $c$, we can arrange that the probability that a square contains at least a Poisson point is as high as we want. Indeed, for all $i$, we have

$$
p \equiv P\left(X\left(s_{i}\right) \geq 1\right)=1-e^{-c^{2}} .
$$

We say that a square is open if it contains at least one point, and closed otherwise. Notice that squares are open (closed) with probability $p$, independently of each other.

We now map this model into a discrete edge-percolation model on the square grid. We draw an horizontal edge across half of the squares, and a vertical edge across the others, as shown on the right-hand side of Fig. 2. In this way we obtain a grid $G_{n}$ of horizontal and vertical edges, each edge being open, independently of all other edges, with probability $p$. We call a path of $G_{n}$ open if it contains only open edges. Note that, for $c$ large enough, our construction produces winding open paths that cross the entire network area, see Fig. 3 for a simulation of 


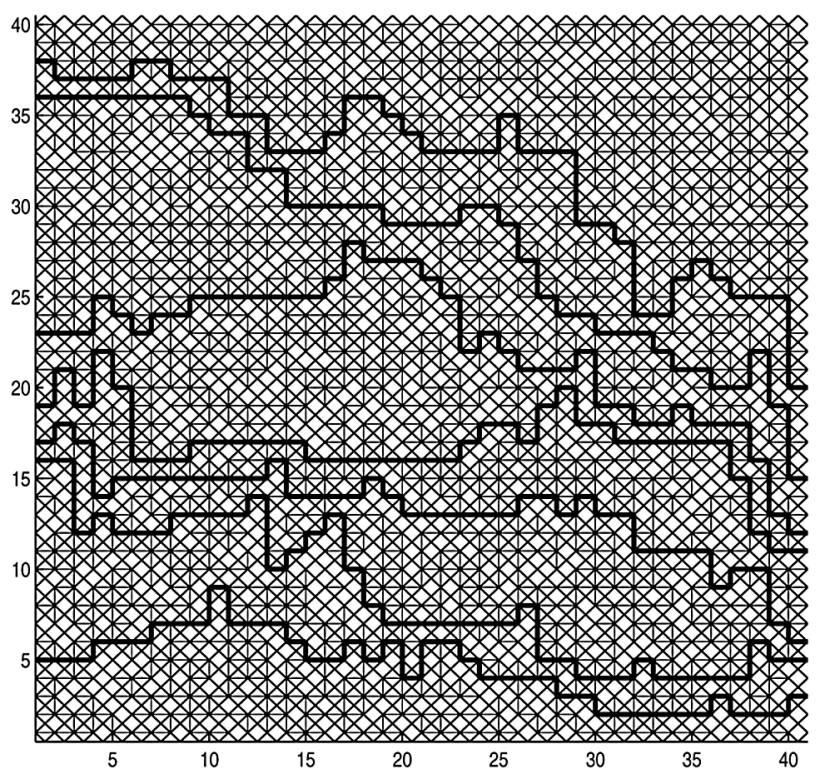

Fig. 3. Horizontal paths in a $40 \times 40$ bond percolation model obtained by computer simulation. Each square is traversed by an open edge with probability $p(p=0.7$ here $)$. Closed edges are not depicted.

this. It is convenient at this point to denote the number of edges composing the side length of the box by $m=\frac{\sqrt{n}}{c \sqrt{2}}$, where $c$ is rounded up such that $m$ is an integer. By Theorem 5 in Appendix I, we can choose $c$ large enough such that there are w.h.p. $\Omega(m)$ paths crossing the network area from left to right, and these can be grouped into disjoint sets of $[\delta \log m\rceil$ paths, each group crossing a rectangle of size $m \times\left(\kappa \log m-\epsilon_{m}\right)$, for all $\kappa>0, \delta$ small enough, and a vanishingly small $\epsilon_{m}$ so that the side length of each rectangle is an integer. See Fig. 8 for a schematic representation of this. The same is true if we divide the area into vertical rectangles and look for paths crossing the area from bottom to top. Using the union bound, we conclude that there exist both horizontal and vertical disjoint paths w.h.p. These paths form a backbone, that we call the highway system.

\section{CAPACITY OF THE HighWAY}

Along the paths of the highway system, we choose one node per edge, that acts as a relay. This is possible as the paths are formed by open edges, which are associated to squares that contain at least one node. The paths are thus made of a chain of nodes such that the distance between any two consecutive nodes is at most $2 \sqrt{2} c$.

To achieve a constant rate along a path, we now divide time into slots. The idea is that when a node along a path transmits, other nodes that are sufficiently far away can simultaneously transmit, without causing excessive interference. The following theorem makes this precise, ensuring that a constant rate $R$, independent of $n$, can be achieved w.h.p. on all the paths simultaneously. The theorem is stated in slightly more general terms considering nodes at $L_{1}$ distance $d$ in the edge percolation grid $G_{n}$, rather than simply neighbors, as this will turn useful again later. Notice that the rate along a crossing path can be immediately obtained by letting $d=1$.

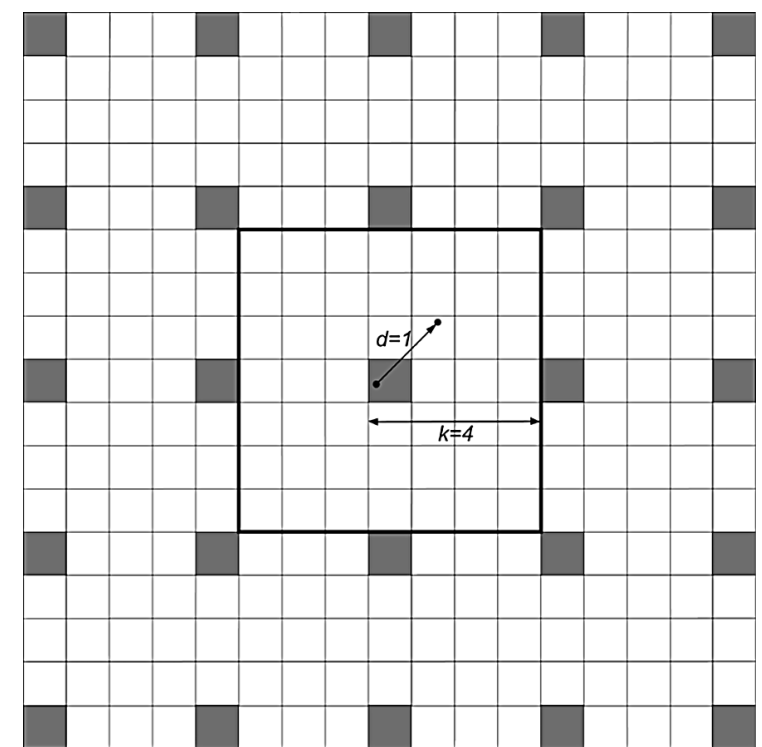

Fig. 4. The situation depicted represents the case $d=1$. Gray squares can transmit simultaneously. Notice that around each grey square there is a "silence" region of squares that are not allowed to transmit in the given time slot.

Theorem 3: For any integer $d>0$, there exist an $R(d)>0$, such that in each square $s_{i}$ there is a node that can transmit w.h.p. at rate $R(d)$ to any destination located within distance $d$. Furthermore, as $d$ tends to infinity, we have

$$
R(d)=\Omega\left(d^{-\alpha-2} e^{-\gamma \sqrt{2} c d}\right)
$$

Proof: We divide time into a sequence of $k^{2}$ successive slots, with $k=2(d+1)$. Then, we consider disjoint sets of subsquares $s_{i}$ that are allowed to transmit simultaneously, as depicted in Fig. 4.

Let us focus on one given subsquare $s_{i}$. The transmitter in $s_{i}$ transmits toward a destination located in a square at distance at most $d$ (diagonal) subsquares away. First, we find an upper bound for the interference at the receiver. We notice that the transmitters in the eight closest subsquares are located at Euclidean distance at least $c(d+1)$ from the receiver, see Fig. 4. The 16 next closest subsquares are at Euclidean distance at least $c(3 d+3)$, and so on. By extending the sum of the interferences to the whole plane, this can then be bounded as follows:

$$
\begin{aligned}
I(d) \leq & \sum_{i=1}^{\infty} 8 i P l(c(2 i-1)(d+1)) \\
\leq & P(c(d+1))^{-\alpha} e^{-\gamma c(d+1)} \\
& \times \sum_{i=1}^{\infty} 8 i(2 i-1)^{-\alpha} e^{-\gamma c(d+1)(2 i-2)}
\end{aligned}
$$

notice that this sum clearly converges if $\gamma>0$ or $\alpha>2$.

Next, we want to bound from below the signal received from the transmitter. We observe first that the distance between the transmitter and the receiver is at most $\sqrt{2} c(d+1)$. Hence, the signal $S(d)$ at the receiver can be bounded by

$$
\begin{aligned}
S(d) & \geq P l(\sqrt{2} c(d+1)) \\
& =P \min \left\{1,(\sqrt{2} c(d+1))^{-\alpha}\right\} e^{-\gamma \sqrt{2} c(d+1)} .
\end{aligned}
$$


Finally, by combining (3) and (4), we obtain a lower bound on the function

$$
R(d)=\log \left(1+\frac{S(d)}{N_{0}+I(d)}\right)
$$

which does not depend on $n$, and hence the first part of the theorem immediately follows.

We now look at the asymptotic behavior of (5) for $d \rightarrow \infty$. Again by combining (3) and (4) and taking the limit for $d \rightarrow \infty$, after some computations it follows easily that

$$
\frac{S(d)}{N_{0}+I(d)}=\Omega\left(d^{-\alpha} e^{-\gamma \sqrt{2} c d}\right)
$$

which also implies that

$$
\log \left(1+\frac{S(d)}{N_{0}+I(d)}\right)=\Omega\left(d^{-\alpha} e^{-\gamma \sqrt{2} c d}\right) .
$$

Finally, accounting for the time division into $k^{2}=4(d+1)^{2}$ time slots, the actual rate available in each square is $\Omega\left(d^{-\alpha-2} e^{-\gamma \sqrt{2} c d}\right)$.

The proof of the following Corollary is immediate by switching the role of transmitters and receivers in the above proof. Distances remain the same, and all equations still hold.

Corollary 1: For any integer $d>0$, there exist an $R(d)>0$, such that in each square $s_{i}$ there is a node that can can receive w.h.p. at rate $R(d)$ from any transmitter located within distance $d$.

Furthermore, as $d$ tends to infinity, we have w.h.p.

$$
R(d)=\Omega\left(d^{-\alpha-2} e^{-\gamma \sqrt{2} c d}\right) .
$$

\section{A. Routing Protocol}

Given the results of the previous section, we can now describe a routing protocol that achieves $\Omega(1 / \sqrt{n})$ per-node rate. The protocol uses four separate phases, and in each phase time is divided into slots. A first phase is used to drain information to the highway, a second one to transport information on the horizontal highways connecting the left and right edges of the domain, a third one to transport information on the vertical highways connecting the top and bottom edges of the domain, and a fourth one to deliver information to the destinations. The draining and delivery phases use direct transmission and multiple time slots, while the highway phases use both multiple hops and multiple time slots. We show that the communication bottleneck is in the highway phase which can achieve a per-node rate of $\Omega(1 / \sqrt{n})$.

We start by proving two simple lemmas that will turn to be useful in the computation of the rate.

Lemma 1: If we partition the box $B_{n}$ into an integer number $m^{2}=\frac{n}{c^{2}}$ of subsquares $s_{i}$ of constant side length $c$, then there are w.h.p. less than $\log m$ nodes in each subsquare.

Proof: The proof follows from Chernoff's bound in Appendix II. Let $A_{n}$ be the event that there is at least one subsquare with more than $\log m$ nodes. Since the number of nodes $\left|s_{i}\right|$ in each subsquare of the partition is a Poisson random variable of parameter $c^{2}$, by the union and Chernoff bounds, we have

$$
\begin{aligned}
P\left(A_{n}\right) & \leq m^{2} P\left(\left|s_{i}\right|>\log m\right) \\
& \leq m^{2} e^{-c^{2}}\left(\frac{c^{2} e}{\log m}\right)^{c^{2} \log m} \\
& =e^{-c^{2}}\left(\frac{c^{2} e^{\frac{2}{c^{2}}+1}}{\log m}\right)^{c^{2} \log m} \rightarrow 0
\end{aligned}
$$

as $m$ tend to infinity.

Lemma 2: If we partition the box $B_{n}$ into an integer number $\frac{\sqrt{n}}{w}$ of rectangles $R_{i}$ of side lengths $\sqrt{n} \times w$, then there are w.h.p. less than $2 w \sqrt{n}$ nodes in each rectangle.

Proof: Again, the proof follows from Chernoff's bound in Appendix II. Let $A_{n}$ be the event that there is at least one rectangle with more than $2 w \sqrt{n}$ nodes. Since the number of nodes $\left|R_{i}\right|$ in each rectangle is a Poisson random variable of parameter $w \sqrt{n}$, by the union and Chernoff bounds, we have

$$
\begin{aligned}
P\left(A_{n}\right) & \leq \frac{\sqrt{n}}{w} P\left(\left|R_{i}\right|>2 w \sqrt{n}\right) \\
& \leq \frac{\sqrt{n}}{w} e^{-w \sqrt{n}}\left(\frac{e w \sqrt{n}}{2 w \sqrt{n}}\right)^{2 w \sqrt{n}} \\
& =\frac{\sqrt{n}}{w} e^{-w \sqrt{n}}\left(\frac{e}{2}\right)^{2 w \sqrt{n}} \rightarrow 0
\end{aligned}
$$

as $n$ tends to infinity.

The next Lemma illustrates the achievable rate in the draining phase of the protocol, occurring in a single hop.

Lemma 3: Every node inside $B_{n}$ can achieve w.h.p. a rate to some node on the highway system of

$$
\Omega\left((\log n)^{-3-\alpha} n^{-\frac{\sqrt{2}}{2} c \kappa \gamma}\right) .
$$

Proof: We want to compute an achievable rate from sources to the highways. Recall that $p=1-e^{-c^{2}}$. By Theorem 5 in Appendix I we can partition the square $B_{n}$ into an integer number of rectangles of size $m \times\left(\kappa \log m-\epsilon_{m}\right)$ and choose $\kappa$ and $c$ such that there are at least $\lceil\delta \log m\rceil$ crossing paths in each rectangle w.h.p. We then slice the network area into horizontal strips of constant width $w$, by choosing $w$ appropriately such that there are at least as many paths as slices inside each rectangle of size $m \times\left(\kappa \log m-\epsilon_{m}\right)$. We can then impose that nodes from the $i$ th slice communicate directly with the $i$ th horizontal path. Note that each path may not be fully contained in its corresponding slice, but it may deviate from it. However, a path is never farther than $\kappa \log m-\epsilon_{m}$ from its corresponding slice.

More precisely, to each source in the $i$ th slab, we assign an entry point on the $i$ th horizontal path. The entry point is defined as the node on the horizontal path closest to the vertical line drawn from the source point, see Fig. 5. The source then transmits directly to the entry point. Theorem 5 and the triangle inequality ensure that the distance between sources and entry points is never larger than $\kappa \log m+\sqrt{2} c$. This is because each 


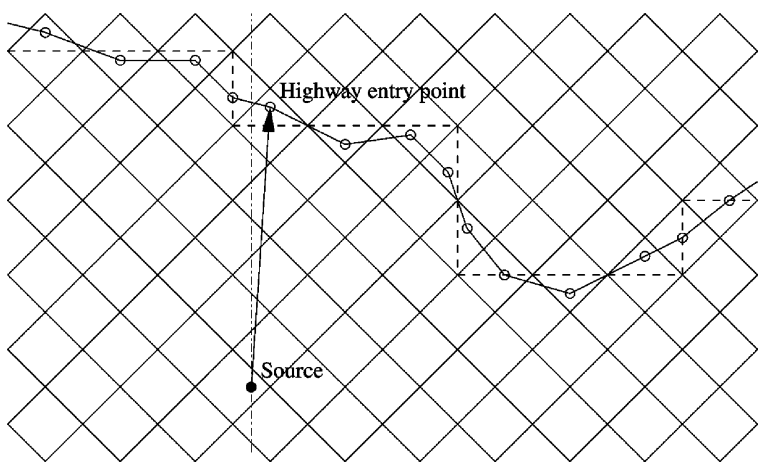

Fig. 5. The draining phase.

rectangle contains $\lceil\delta \log m\rceil$ paths, and therefore each source finds its highway within the same rectangle.

Hence, to compute the rate at which nodes can communicate to the entry points, we let $d=\kappa \log m+\sqrt{2} c$ and apply the second part of Theorem 3 . We obtain that one node per square can communicate to its entry point at rate

$$
\begin{aligned}
R(\kappa \log m+\sqrt{2} c) & =R\left(\kappa \log \frac{\sqrt{n}}{\sqrt{2} c}+\sqrt{2} c\right) \\
& =\Omega\left(\frac{e^{-\gamma \sqrt{2} c \kappa \log \frac{\sqrt{n}}{\sqrt{2} c}}}{\left(\kappa \log \frac{\sqrt{n}}{\sqrt{2} c}\right)^{2+\alpha}}\right) \\
& =\Omega\left(\frac{n^{-\frac{\sqrt{2}}{2} c \kappa \gamma}}{(\log n)^{2+\alpha}}\right) .
\end{aligned}
$$

Now we note that as there are possibly many nodes in the squares, they have to share this bandwidth. Using Lemma 1, we conclude that the transmission rate of each node in the draining phase of our protocol is at least $R(d) / \log m$, which concludes the proof.

The following lemma illustrates the achievable rate on the multihop routes along the highway.

Lemma 4: The nodes along the highways can achieve w.h.p. a per-node rate of $\Omega\left(\frac{1}{\sqrt{n}}\right)$.

Proof: We divide horizontal and vertical information flow, adopting the following multihop routing policy: pairwise coding and decoding is performed along horizontal highways, until we reach the crossing with the target vertical highway. Then, the same is performed along the vertical highways until we reach the appropriate exit point for delivery.

We start by considering the horizontal traffic. Let a node be sitting on the $i$ th horizontal highway and compute the traffic that goes through it. Notice that, at most, the node will relay all the traffic generated in the $i$ th slice of width $w$.

According to Lemma 2, a node on a horizontal highway must relay traffic for at most $2 w \sqrt{n}$ nodes. As the maximal distance between hops is constant, by applying Theorem 3 we conclude that an achievable rate along the highways is $\Omega(1 / \sqrt{n})$, with high probability.

The problem of the vertical traffic is the dual of the previous one. We can use the same arguments to compute the receiving rate of the nodes. Since each node is the destination of exactly one source, the rate per node becomes the same as above.
The following Lemma illustrates the achievable rate in the receiving phase of the protocol, occurring in a single hop.

Lemma 5: Every destination node can receive w.h.p. information from the highway at rate $\Omega\left((\log n)^{-\alpha-3} n^{-\frac{\sqrt{2}}{2} c \kappa \gamma}\right)$.

Proof: The delivery phase consists in communicating from the highway system to the actual destination. We proceed exactly in the same way as in Lemma 3, but in the other direction, that is, horizontal delivery from the vertical highways.

We divide the network area into vertical slices of constant width, and define a mapping between slabs and vertical paths. We assume that communication occurs from an exit point located on the highway, which is defined as the node of the vertical path closest to the horizontal line drawn from the destination. Again, the distance between exit points and destination is at most $\kappa \log m+\sqrt{2} c$. We can thus let $d=\kappa \log m+\sqrt{2} c$ in Corollary 1 , and conclude that each square can be served at rate

$$
R(d)=\Omega\left(\frac{n^{-\frac{\sqrt{2}}{2} c \kappa \gamma}}{(\log n)^{2+\alpha}}\right) .
$$

As there are at most $\log m$ nodes in each square by Lemma 1 , the rate per node is at least equal to $R(d) / \log m$.

We are finally ready to provide a proof of Theorem 1 .

Proof: We observe by Lemmas 3, 4, and 5, that if

$$
\frac{\sqrt{2}}{2} c \kappa \gamma<\frac{1}{2}
$$

then the overall per-node rate is limited by the highway phase only, and the proof follows immediately from Lemma 4. Hence, we have to make sure that we can satisfy (7). Recall that $p=$ $1-e^{-c^{2}}$ and by Theorem 5 that $c$ and $\kappa$ are constrained to be such that

$$
c^{2}>\log 6+\frac{2}{\kappa} .
$$

From (7) and (8) it follows that we can choose $\kappa=\frac{1}{2 \sqrt{2} c \gamma}$ and $c>\left(2 \sqrt{2} \gamma+\sqrt{8 \gamma^{2}+\log 6}\right)$ to conclude the proof of the first part of the theorem.

As for the second part of the theorem, we need to bound the number of hops needed to reach the destination with the described protocol. For any source-destination pair, the number of hops is given by two plus the number of hops on the highway, which is at most twice the number of hops of a crossing path between the shortest sides of a rectangle of size $m \times \kappa \log m$. Recall now that in each rectangle there are w.h.p. at least $\delta \log m$ disjoint crossing paths between its shortest sides, and at most $\kappa m \log m$ edges. Letting $N_{m}$ be the total number of hops along all crossing paths inside a rectangle and $l(i)$ be the number of hops of path $i$, we have by taking the expectation with respect to the network realization

$$
E\left(N_{m}\right) \leq E\left(\sum_{i=1}^{\delta \log m} l(i)\right) \leq \kappa m \log m .
$$


By taking the expectation inside the sum, it now follows that the average number of hops to reach the destination is at most proportional to $m=\frac{\sqrt{n}}{c \sqrt{2}}$.

\section{DENSE NETWORKS}

In this section, we consider the model where nodes are distributed according to a Poisson point process of intensity $n$ over a square of unit area. Furthermore, we take an attenuation function $l$ of the form

$$
l(d)=d^{-\alpha} e^{-\gamma d} .
$$

In this case, we divide that network into squares of size $c / \sqrt{n}$. We obtain thus the same number of little squares as in the previous model. The average number of nodes in each little square is also the same, namely $c^{2}$. Therefore, all the percolation results above still hold for this model, and we can find as many highways as above.

To derive the lower bound on the capacity, we have to compute the throughput along the highways, as well as the rate at which nodes can send data toward the highways. Both of these throughputs were computed using Theorem 3, so it is enough here to give an adapted version of such theorem.

Theorem 4: For any integer $d>0$, there exist an $R(d)>0$, such that in each square $s_{i}$ there is a node that can transmit w.h.p. at rate $R(d)$ to any destination located within distance $d$. Furthermore, as $d$ tends to infinity, we have

$$
R(d)=\Omega\left(d^{-2}\right) .
$$

Proof: We set up the same time division scheme as in Theorem 3 , with $k^{2}$ time slots, where $k=2(d+1)$. Similarly, the 8 closest interferers are located at least $d+2$ squares away from the receiver, the next 16 interferers at distance $3 d+3$, and so on. The difference here is that now squares have size $c / \sqrt{n}$. The sum of the interferences at the receiver can be bounded as follows:

$$
\begin{aligned}
I(d, n) \leq & \sum_{i=1}^{\infty} 8 i P l\left(\frac{c(2 i-1)(d+1)}{\sqrt{n}}\right) \\
= & \sum_{i=1}^{\infty} 8 i P\left(\frac{c(2 i-1)(d+1)}{\sqrt{n}}\right)^{-\alpha} e^{-\gamma c(2 i-1) \frac{(d+1)}{\sqrt{n}}} \\
\leq & P\left(\frac{c(d+1)}{\sqrt{n}}\right)^{-\alpha} e^{-\gamma c \frac{(d+1)}{\sqrt{n}}} \\
& \sum_{i=1}^{\infty} 8 i(2 i-1)^{-\alpha} e^{-\gamma c(2 i-2) \frac{(d+1)}{\sqrt{n}}}
\end{aligned}
$$

This sum clearly converges if $\alpha>2$ or $\gamma>0$.

As the receiver is at most $d$ squares away from the transmitter, the Euclidean distance between them is less than $c \sqrt{2}(d+1) / \sqrt{n}$. The strength $S(d, n)$ of the signal at the receiver is thus

$$
S(d, n) \geq P\left(\frac{c \sqrt{2}(d+1)}{\sqrt{n}}\right)^{-\alpha} e^{-\gamma c \sqrt{2} \frac{(d+1)}{\sqrt{n}}} .
$$

We now take the limit of the signal to noise plus interference ratio for $n \rightarrow \infty$. By combining (10) and (11) one obtains a constant bound, independent of $d$

$$
\lim _{n \rightarrow \infty} \operatorname{SNIR}(d, n)=\lim _{n \rightarrow \infty} \frac{S(d, n)}{N_{0}+I(d, n)} \geq K .
$$

This means that a constant rate is achievable w.h.p. in each time slot. This proves the first part of the theorem.

The same computation shows that if $d$ increases with $n$, the above limit still holds whenever $(d+1) / \sqrt{n}$ tends to zero. Therefore, if $d=O(\sqrt{n})$, a constant rate is achievable w.h.p. for each active transmission. However, as there are $k^{2}=4(d+$ $1)^{2}$ time slots in our TDMA scheme, the actual throughput available for each square must be divided by $k^{2}$, and thus

$$
R(d)=\Omega\left(d^{-2}\right) .
$$

\section{CONCLUSION}

We have found that the capacity of wireless networks of randomly located nodes has the same asymptotic behavior as the capacity of arbitrary networks: nodes in a random network can transmit at the same rate as nodes in an arbitrary network and there is no price to pay-at least asymptotically-for the additional randomness present in the system. This result closes a previous gap between upper and lower bounds on the optimal per-node transmission rate that consistently appeared in different proofs proposed in the literature.

We were able to close this gap by exploiting a connection between percolation theory and the way we scale the transmission ranges of the nodes. By scaling the ranges at a sufficiently slow rate, a wireless backbone containing paths that cross the network area exists with high probability, but this covers all the nodes, generating excessive interference. By scaling the ranges at a higher rate, this backbone does not form at all. Previous results used the first kind of scaling to prove a capacity lower bound. We noticed that percolation theory ensures that a different kind of backbone forms in the transition region between this two extreme scalings. This does not cover all the nodes, nevertheless it is sufficiently rich in crossing paths so that it can transport the total amount of traffic. By operating the network in this transition region between order and disorder, we are able to prove our tight bound. Interestingly, the choice of the adopted scaling law depends on the physical attenuation inside the medium where wireless propagation takes place.

Finally, we point out that the problem of optimizing the throughput pre-constant remains open, as well as extensions to models which take random fading into account.

\section{APPENDIX I \\ PERCOLATION THEORY RESUlts}

In this section, we build the percolation theory background that is needed to show the existence of a cluster of nodes forming the highway system. We consider the independent bond percolation model on the square lattice. That is, we declare each edge of an infinite square grid open with probability $p$ and closed otherwise, independently of all other edges. and let $B_{m}$ denote a 
box of side length $m$ embedded in the square lattice. We denote by $B_{m}^{\leftrightarrow}$ the event that there is a crossing path connecting the left side of $B_{m}$ with its right side. We need a series of results that culminate with Theorem 5 below. The proofs of the first two propositions follow standard arguments, and are reported for completeness.

Proposition 1: For $p<\frac{1}{3}$ and for all $m$, we have

$$
P_{p}\left(B_{m}^{\leftrightarrow}\right) \leq \frac{4}{3}(m+1) e^{-\alpha(p) m}
$$

where $\alpha(p)=-\log 3 p$.

Proof: Let $B_{2 m}$ be a box of side length $2 m$ centered at the origin of the square lattice, and $0 \leftrightarrow \partial B_{2 m}$ be the event that there is a path connecting the origin to the boundary of $B_{2 m}$. Denoting by $N(m)$ the number of open paths of length $m$ starting at the origin

$$
P_{p}\left(0 \leftrightarrow \partial B_{2 m}\right) \leq P_{p}(N(m) \geq 1)
$$

since a path connecting the origin to the boundary of $B_{2 m}$ has length at least $m$. As paths of length $m$ are open with probability $p^{m}$, we can bound this probability by

$$
P_{p}(N(m) \geq 1) \leq p^{m} \sigma(m)
$$

where $\sigma(m)$ denotes the number of paths of length $m$ starting at the origin. This number is obviously not larger than

$$
\sigma(m) \leq 4 \cdot 3^{m-1}
$$

Combining these three inequalities we have

$$
P_{p}\left(0 \leftrightarrow \partial B_{2 m}\right) \leq \frac{4}{3}(3 p)^{m}=\frac{4}{3} e^{-\alpha(p) m},
$$

where $\alpha(p)=-\log 3 p$.

Let us now order the vertices on the left side of the box $B_{m}$ starting from the bottom, and let $C_{i}$ be the event that there exist a crossing path starting from the $i$ th vertex. There is a nonrandom index $i_{0}$ so that

$$
P_{p}\left(C_{i_{0}}\right) \geq \frac{1}{m+1} P_{p}\left(B_{m}^{\leftrightarrow}\right)
$$

Now choose the box $B_{m}$ with this $i_{0}$ th vertex being at the origin; see Fig. 6 for an illustration of this construction with $i_{0}=3$. We then write

$$
\begin{aligned}
P_{p}\left(B_{m}^{\leftrightarrow}\right) & \leq(m+1) P_{p}\left(C_{i_{0}}\right) \\
& \leq(m+1) P_{p}\left(0 \leftrightarrow \partial B_{2 m}\right) \\
& \leq \frac{4}{3}(m+1) e^{-\alpha(p) m}
\end{aligned}
$$

The proposition above has shown that when $p$ is small enough the box $B_{m}$ cannot be crossed from left to right. We now turn to the dual question of the existence of a crossing path when $p$ is sufficiently high. The following result exploits the concept of the dual lattice that is often used in percolation theory. The

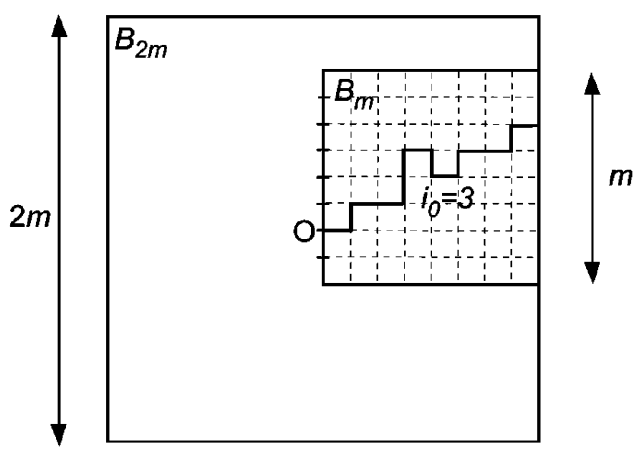

Fig. 6. The probability of crossing the box $B_{m}$ starting from the third vertex from the bottom of $B_{m}$ is less than the probability of reaching the boundary of box $B_{2 m}$ starting from its center.

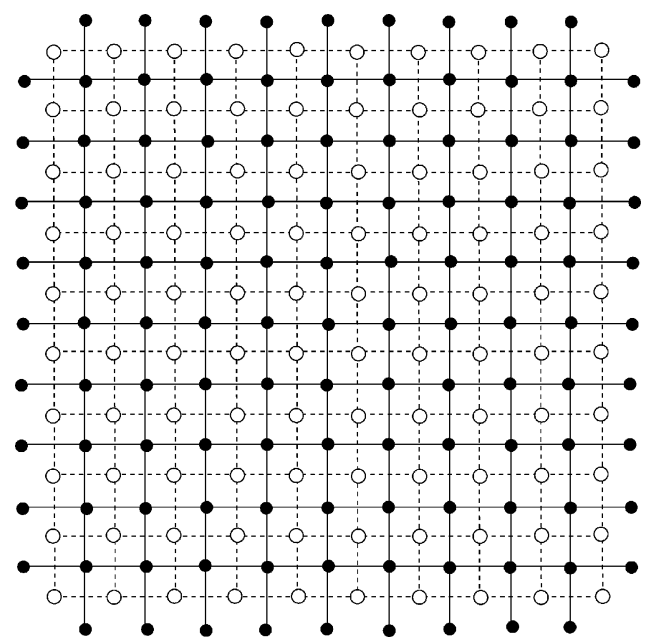

Fig. 7. The box $B_{m}$ is drawn with a continuous line, the dual box $S_{m}$ is drawn with a dashed line. Whenever there is not a top to bottom crossing in the $S_{m}$, then there must be a left to right crossing in $B_{m}$.

dual lattice is defined by placing a vertex in each square of the lattice, and joining two such vertices by an edge whenever the corresponding squares share a side, see Fig. 7. We can also construct a dual of the random grid by drawing an edge in the dual lattice, if it does not cross an edge of the original random grid, and deleting it otherwise.

Proposition 2: For $p>\frac{2}{3}$ and for all $m$, we have

$$
P_{p}\left(B_{m}^{\leftrightarrow}\right) \geq 1-\frac{4}{3}(m+1) e^{-\alpha(1-p) m}
$$

where $\alpha(\cdot)$ is as before.

Proof: Let us consider the box $B_{m}$, and the corresponding dual box $S_{m}$ as depicted in Fig. 7. Let $A_{m}$ be the event that there is a left to right crossing path of $B_{m}$. Its complement $A_{m}^{c}$ is the event that there exists a top to bottom crossing path in $S_{m}$. This last statement, which is immediate by inspection of Fig. 7, is given a complete topological proof in [12].

By rotating the box by $90^{\circ}$ and applying Proposition 1 to the dual lattice, we have that, for all $m$

$$
P\left(A_{m}^{c}\right) \leq(n+1) \frac{4}{3} e^{-\alpha(1-p) m}
$$

The result now follows immediately. 


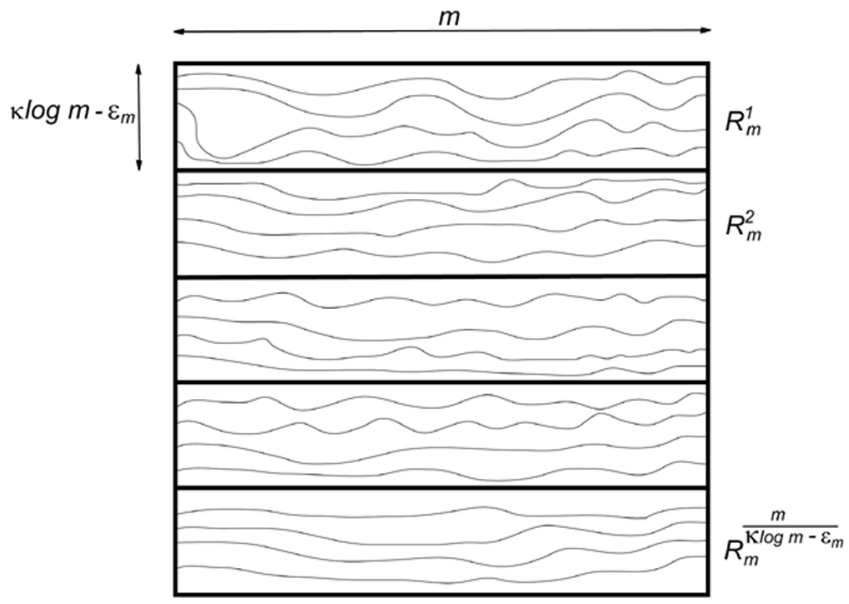

Fig. 8. There exists a large number of crossing paths in $B_{m}$ that behave almost as straight lines.

We now turn to the question of how many crossing paths there are when $p$ is sufficiently high. To show the existence of many disjoint crossing paths, we need one more result, that is [9, Theorem 2.45]. We state this result next and refer to [9] for a proof. We define an event $A$ to be increasing if adding an edge in any realization of the random network where $A$ occurs, leads to a configuration which is still in $A$. Notice that the event of having a crossing from the left to the right side of $B_{m}$ is an increasing event. Next we define $I_{r}\left(A_{m}\right)$ to be the event defined by the set of configurations in $A$ for which $A$ remains true even if we change the states of up to $r$ arbitrary edges. A little thinking reveals that this is just the event that $r+1$ edge-disjoint crossings exist. More formally, one can refer to the max-flow min-cut theorem to show this, see for example, [19]. The set $I_{r}(A)$ is sometimes called the interior of $A$ of depth $r$. We have the following lemma.

Lemma 6: Let $A$ be an increasing event, and let $I_{r}(A)$ be as defined above. We have

$$
1-P_{p_{2}}\left(I_{r}(A)\right) \leq\left(\frac{p_{2}}{p_{2}-p_{1}}\right)^{r}\left(1-P_{p_{1}}(A)\right)
$$

for any $0 \leq p_{1}<p_{2} \leq 1$.

Given the results above we can now show the existence of many disjoint crossing paths. It turns out that for all $\kappa>0$, if we divide the box into rectangular slices of side length $m \times$ $\kappa \log m$, then if we choose $p$ sufficiently high, each of these rectangles contains at least a constant times $\log m$ number of disjoint crossings between the two shortest sides. This means that not only there exist a large number of crossings of $B_{m}$, but also that these crossings behave almost as straight lines in connecting the two sides of the box, as they do not "wiggle" more than a $\kappa \log m$ amount.

More formally, for any given $\kappa>0$, let us partition $B_{m}$ into rectangles $R_{m}^{i}$ of sides $m \times\left(\kappa \log m-\epsilon_{m}\right)$, see Fig. 8 . We choose $\epsilon_{m}>0$ as the smallest value such that the number of rectangles $\frac{m}{\kappa \log m-\epsilon_{m}}$ in the partition is an integer. It is easy to see that $\epsilon_{m}=o(1)$ as $m \rightarrow \infty$. We let $C_{m}^{i}$ be the maximal number of edge-disjoint left to right crossings of rectangle $R_{m}^{i}$ and let $N_{m}=\min _{i} C_{m}^{i}$. The result is the following.

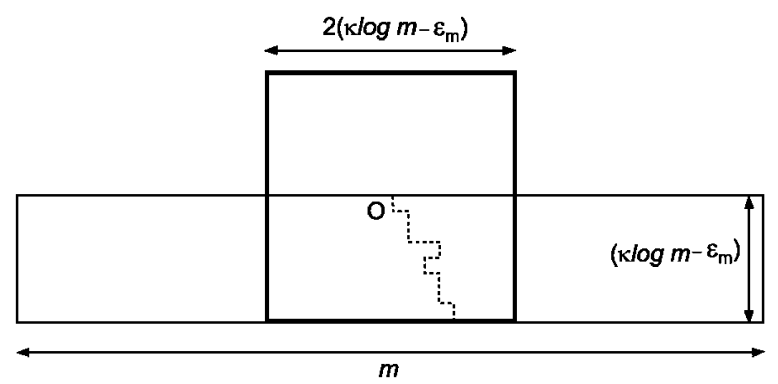

Fig. 9. Crossing the rectangle from left to right implies that there cannot be a top to bottom crossing in the dual graph. Hence, there cannot be a path from the center to the boundary of any of the dual of the $m+1$ squares centered on the upper boundary of the rectangle.

Theorem 5: For all $\kappa>0$ and $\frac{5}{6}<p<1$ satisfying $2+$ $\kappa \log (6(1-p))<0$, there exists a $\delta(\kappa, p)>0$ such that

$$
\lim _{m \rightarrow \infty} P_{p}\left(N_{m} \leq \delta \log m\right)=0 .
$$

Proof: Let $R_{m}^{i \leftrightarrow}$ be the event that there exists a left to right crossing of rectangle $R_{m}^{i}$. With reference to Fig. 9, for all $p>\frac{2}{3}$, and so in particular for $p>\frac{5}{6}$, we have

$$
\begin{aligned}
P\left(R_{m}^{i \leftrightarrow}\right) \geq & 1-(m+1) P\left(0 \leftrightarrow \partial B_{2\left(\kappa \log m-\epsilon_{m}\right)}\right) \\
\geq & 1-\frac{4}{3}(m+1) e^{-\left(\kappa \log m-\epsilon_{m}\right)(-\log (3(1-p)))} \\
= & 1-\frac{4}{3}(m+1) m^{\kappa \log (3(1-p))} \\
& \times(3(1-p))^{-\epsilon_{m}}
\end{aligned}
$$

where the first inequality follows from the same argument as in the proof of Proposition 2 and should be clear by looking at Fig. 9, and the last inequality follows from Proposition 1 applied to the dual lattice. We now use the increment trick of Lemma 6 to obtain $r=\delta \log m$ such crossings in $R_{m}^{i}$. For all $\frac{2}{3}<p^{\prime}<$ $p<1$, by Lemma 6 and (12), we have

$$
\begin{aligned}
P_{p}\left(C_{m}^{i} \leq \delta \log m\right) \leq & \left(\frac{p}{p-p^{\prime}}\right)^{\delta \log m} \\
& \times \frac{4}{3}(m+1) m^{\kappa \log \left(3\left(1-p^{\prime}\right)\right)} \\
& \times\left(3\left(1-p^{\prime}\right)\right)^{-\epsilon_{m}} .
\end{aligned}
$$

Since $p>\frac{5}{6}$, we have, by letting $p^{\prime}=2 p-1>\frac{2}{3}$

$$
\begin{aligned}
& P_{p}\left(C_{m}^{i} \leq \delta \log m\right) \leq \frac{4}{3}(m+1) m^{\delta \log \frac{p}{1-p}+\kappa \log (6(1-p))} \\
& \times(6(1-p))^{-\epsilon_{m}}
\end{aligned}
$$

We finally consider the probability of having at most $\delta \log m$ edge-disjoint left to right crossings in every rectangle $R_{m}^{i}$. By independence and using (14), we have

$$
\begin{aligned}
P_{p}\left(N_{m} \leq \delta \log m\right)= & \left(P_{p}\left(C_{m}^{i} \leq \delta \log m\right)\right)^{\frac{m}{\log m-\epsilon_{m}}} \\
\leq & \left(\frac{4}{3}(m+1) m^{\delta \log \frac{p}{1-p}+\kappa \log 6(1-p)}\right. \\
& \left.\times(6(1-p))^{-\epsilon_{m}}\right)^{\frac{m}{\log m-\epsilon_{m}}} .
\end{aligned}
$$


Since $\epsilon_{m}=o(1)$, we have that (15) tends to zero if

$$
\delta \log \frac{p}{1-p}+1+\kappa \log (6(1-p))<-1 .
$$

To complete the proof we can choose $\delta(\kappa, p)$ small enough so that (16) is satisfied.

\section{APPENDIX II \\ CHERnOFF BOUnd FOR A POISSON RANDOM VARIABLE}

We have used the following Chernoff bound for a Poisson random variable $X$ of parameter $\lambda$

$$
P(X \geq x) \leq \frac{e^{-\lambda}(e \lambda)^{x}}{x^{x}}, \quad \text { for } x>\lambda
$$

Proof:

$$
\begin{aligned}
E\left(e^{s X}\right) & =\sum_{k=0}^{\infty} \frac{e^{-\lambda} \lambda^{k}}{k !} e^{s k} \\
& =e^{\lambda\left(e^{s}-1\right)} \sum_{k=0}^{\infty} \frac{e^{-\lambda e^{s}}\left(\lambda e^{s}\right)^{k}}{k !} \\
& =e^{\lambda\left(e^{s}-1\right)} .
\end{aligned}
$$

For any $s>0$ and $x>\lambda$ applying Markov inequality we have

$$
P(X \geq x) \leq \frac{E\left(e^{s X}\right)}{e^{s x}}=e^{\lambda\left(e^{s}-1\right)-s x} .
$$

Letting $s=\ln (x / \lambda)>0$ we finally obtain

$$
P(X \geq x) \leq e^{x-\lambda-x \ln (x / \lambda)}=\frac{e^{-\lambda}(e \lambda)^{x}}{x^{x}}
$$

\section{REFERENCES}

[1] L. Booth, J. Bruck, M. Franceschetti, and R. Meester, "Covering algorithms, continuum percolation, and the geometry of wireless network," Ann. Appl. Probab., vol. 13, no. 2, pp. 722-731, 2003.

[2] S. R. Broadbent and J. M. Hammersley, "Percolation processes I. Crystals and mazes," in Proc. Cambridge Philosoph. Soc., 1957, vol. 53, pp. 629-641.

[3] O. Dousse, F. Baccelli, and P. Thiran, "Impact of interferences on connectivity of ad hoc networks," ACM/IEEE Trans. Netw., vol. 13, no. 2, pp. 425-436.

[4] O. Dousse and P. Thiran, "Connectivity vs capacity in dense ad hoc networks," in Proc. IEEE Inf. Commun. Conf., INFOCOM 2004.

[5] A. E. Gamal, J. Mammen, B. Prabhakar, and D. Shah, "Throughput delay trade-off in wireless networks," in Proc. IEEE Inf. Commun. Conf., INFOCOM 2004..

[6] M. Franceschetti, L. Booth, M. Cook, R. Meester, and J. Bruck, "Continuum percolation with unreliable and spread out connections," J. Statist. Phys., vol. 118, no. 3-4, pp. 719-731.

[7] M. Franceschetti, J. Bruck, and L. Schulman, "A random walk model of wave propagation," IEEE Trans. Antennas Propag., vol. 52, pp. 1304-1317, May 2004.

[8] E. N. Gilbert, "Random plane networks," J. SIAM, vol. 9, pp. 533-543, 1961.

[9] G. Grimmett, Percolation, Second ed. New York: Springer-Verlag, 1999.

[10] P. Gupta and P. R. Kumar, "The capacity of wireless networks," IEEE Trans. Inf. Theory, vol. 46, pp. 388-404, 2000.

[11] A. Jovicic, P. Viswanath, and S. R. Kulkarni, "Upper bounds to transport capacity of wireless networks," IEEE Trans. Inf. Theory, vol. 50, pp. 2555-2565, 2004.

[12] H. Kesten, Percolation Theory for Mathematicians. Boston, MA: Birkhäuser, 1982.

[13] S. Kulkarni and P. Viswanath, "A deterministic approach to throughput scaling in wireless networks," IEEE Trans. Inf. Theory, vol. 50, pp. 1041-1049, 2004.

[14] O. Lévêque and E. Telatar, "Upper bounds on the capacity of ad-hoc wireless networks," IEEE Trans. Inf. Theory, vol. 51, pp. 858-865.

[15] R. Meester and R. Roy, Continuum Percolation. Cambridge, U.K.: Cambridge Univ. Press, 1996.

[16] T. K. Philips, S. S. Panwar, and A. N. Tantawi, "Connectivity properties of a packet radio network model," IEEE Trans. Inf. Thoery, vol. 35, pp. 1044-1047, 1989.

[17] S. Toupis and A. J. Goldsmith, "Large wireless networks under fading, mobility, and delay constraints," in Proc. IEEE Inf. Commun. Conf., INFOCOM 2004.

[18] L. L. Xie and P. R. Kumar, "A network information theory for wireless communication: Scaling laws and optimal operation," IEEE Trans. Inf. Theory, vol. 50, pp. 748-767, 2004.

[19] R. J. Wilson, Introduction to Graph Theory. London, U.K.: Longman, 1979. 